\title{
The Relation Between Atrial Fibrillation Wavefront Characteristics and Accessory Pathway Conduction
}

\author{
James J.C. Ong, ${ }^{*}$ Yong-Mei Cha, ${ }^{\ddagger}$ Jolene M. Kriett, ${ }^{\S}$ Ker Boyce, $"$ Gregory K. Feld, ${ }^{\ddagger}$ and Peng-Sheng Chen ${ }^{*}$ \\ *Division of Cardiology, Department of Medicine, Cedars-Sinai Medical Center, and the School of Medicine, UCLA, Los Angeles, \\ California 90048; ${ }^{\ddagger}$ Division of Cardiology, Department of Medicine, and ${ }^{\S}$ Division of Cardiothoracic Surgery, Department of Surgery, \\ UCSD Medical Center; and "Division of Cardiology, Department of Medicine, Naval Hospital, San Diego, California 92103
}

\begin{abstract}
Although the source-sink relationship for impulse propagation in cardiac tissues has been demonstrated in vitro, there has been no verification of this hypothesis in humans. Accordingly, eight patients undergoing surgical division of their accessory pathways were studied. A 56-channel $(7 \times 8)$ bipolar plaque electrode array was placed over the atrioventricular groove on the accessory pathway and atrial fibrillation electrically induced. 10 episodes of QRS transition from consecutively preexcited to nonpreexcited complexes were analyzed. This showed that consecutively preexcited QRS complexes were always associated with uniform large atrial wavefronts. Immediately prior to QRS transition, four general types of changes were observed: $(a)$ premature invasion by secondary wavefronts creating local conduction block ( $n$ $=5)$; $(b)$ wavefront collision leading to wavefront curvature $(n=2)$; (c) transition from a uniform large atrial wavefront to multiple fractionated small wavefronts $(n=1)$; and $(d)$ uniform atrial wavefronts "marching" into the accessory pathway refractory period $(n=2)$. We conclude that local atrial wavefront characteristics are important factors influencing impulse propagation through the accessory pathway. The findings that local wavefront collision, curvature, or fractionation often precede loss of accessory pathway conduction support the notion that source-sink relationship is an important determinant of the safety factor for impulse propagation in the human heart. (J. Clin. Invest. 1995. 96:2284-2296.) Key words: Wolff-Parkinson-White syndrome • electrophysiology • source-sink relationship • impulse propagation $\cdot$ safety factor
\end{abstract}

\section{Introduction}

Safety factor of impulse propagation in cardiac tissue depends on the interaction between the amount of current available up-

This paper was presented in part in abstract form at the 67th Scientific Session of the American Heart Association, Dallas, Texas on 14-17 November 1994.

Address correspondence to James J.C. Ong, M.D., Section of Electrophysiology, Division of Cardiology, Cedars-Sinai Medical Center, Room 5342, 8700 Beverly Boulevard, Los Angeles, CA 90048. Phone: 310-855-4851, FAX: 310-289-0780.

Received for publication 15 February 1995 and accepted in revised form 8 August 1995.

J. Clin. Invest.

(C) The American Society for Clinical Investigation, Inc.

$0021-9738 / 95 / 11 / 2284 / 13 \$ 2.00$

Volume 96, November 1995, 2284-2296 stream (the source) and the structure which determines current density downstream (the sink). The importance of this sourcesink relationship in determining cardiac impulse propagation has been amply demonstrated in the in vitro preparations. For example, Mendez et al. (1) reported in the dog heart that the margin of safety for impulse propagation in the muscle to Purkinje direction is higher than in the opposite direction. The authors proposed that the Purkinje-muscle junction was a funnel like system whose narrow portion corresponded to a terminal Purkinje fiber and whose conical portion composed of increasing number of interconnected muscle fibers. The source-sink relationship of this funnel predicts that it is easier for an impulse to travel from muscle to Purkinje fibers than in the opposite direction. This funnel hypothesis was later applied to a model of preexcitation syndrome by de la Fuente and colleagues (2), who studied the conduction between two pieces of canine atrial tissue connected by a narrow isthmus. The authors demonstrated that conduction block usually occurred at the junction where the small isthmus inserts into the larger piece of atrial tissue, where the source is small and the sink is large. Using a similar model, Cabo et al. (3) recently studied the importance of the source-sink relationship as a cause of conduction block. Narrow isthmuses were created in square pieces of isolated sheep epicardial muscle, and the propagation of impulses through these isthmuses were observed by an optical mapping system. The authors found that the width of the isthmus was important in determining the success or failure of impulse propagation. However, if the width of the isthmus was fixed, propagation through the isthmus was rate dependent. Faster pacing rates decreased the safety factor of propagation through the isthmus, while slower rates favored conduction. The authors proposed that this source-sink relationship may underlie the fundamental mechanisms of functional reentry, and was therefore clinically important.

While the source-sink relationship has been well studied in animal tissues in vitro, whether or not the same relationship is important in the manifestations of clinical arrhythmias remains undefined. One ideal model to test the source-sink relationship in humans is the preexcitation syndrome, where cardiac impulses naturally propagate from the atria to the ventricles via a narrow isthmus, the accessory pathway. During sinus rhythm or atrial fibrillation with antegrade accessory pathway conduction, the atrium serves as the source for the accessory pathway, which in turn acts as the source for the larger ventricle. We hypothesized that because the width of the accessory pathway is fixed, the source-sink relationship would predict that the propagation of atrial impulses through the accessory pathway into the ventricle is dependent on the characteristics of atrial wavefronts arriving at the accessory pathway. Specifically, slower and spatially more uniform atrial wavefronts, providing a larger source current, should favor accessory pathway conduc- 
tion. On the other hand, faster and spatially less uniform atrial wavefronts, providing a smaller source current, should favor conduction block. The purpose of this study was to examine the relation between local atrial wavefront characteristics and accessory pathway conduction during atrial fibrillation to test the source-sink hypothesis in humans.

\section{Methods}

The data in this study were acquired before the era of radiofrequency ablation, when surgical division of the accessory pathway was the standard method of treatment for patients with drug refractory preexcitation syndrome. Eight patients with at least one left free wall accessory pathways were studied during surgery. Informed consents were obtained in all patients in accordance with the institutional review board and all patients underwent routine pre-operative electrophysiological studies.

The surgical technique has been previously described (4). Briefly, a median sternotomy was made and the heart was suspended in a pericardial cradle. Bipolar epicardial wires were placed in the atrium and the ventricle for programmed stimulation. A sock or band electrode array was placed over the atrioventricular groove and data were acquired and analyzed with a 64-channel Computerized Mapping System (Bard Electrophysiology, Tewksbury, MA). The sites of the earliest ventricular activation on the isochronal activation map were identified during atrial pacing with maximum preexcitation. The sites of the earliest atrial activation were identified during orthodromic reciprocating tachycardia or ventricular pacing.

Study protocol. Once the accessory pathway was localized, a research plaque electrode array was placed over the accessory pathway at the atrioventricular junction. This electrode array consisted of 40 ( 5 $\times 8)$ bipolar electrodes in one patient and $56(7 \times 8)$ bipolar electrodes in the remaining seven patients. The inter-electrode distance (distance between bipolar pairs) was $5 \mathrm{~mm}$ and the inter-polar distance was $0.5 \mathrm{~mm}$. Atrial fibrillation was then induced with rapid atrial pacing. Simultaneous local atrial, accessory pathway, and ventricular activations were recorded with the electrode array using a filter setting of 30-300 $\mathrm{Hz}$ at a sampling rate of 1,000 per second. Simultaneous surface ECG were also recorded (leads I, II, III, AVR, AVL, AVF). Eight seconds of data were obtained and stored on a computer disk for later off-line analysis.

Data analysis. 10 episodes of QRS transition from consecutively preexcited to nonpreexcited morphology were analyzed to determine the mechanism(s) leading to loss of accessory pathway conduction. For each activation complex, the time of the greatest $\mathrm{dv} / \mathrm{dt}$ was selected to be the time of local epicardial activation. Isochronal maps were drawn for each atrial wavefront arriving at the accessory pathway. Whether these wavefronts resulted in activation of the accessory pathway was determined by the following criteria and definitions:

Antegrade accessory pathway activation was judged to have occurred if the accessory pathway ventricular insertion site showed the earliest ventricular activation, followed by a centrifugal spread of activation from that point (Fig. 1, $A$ and $C$ ). In contrast, a non-preexcited beat with activation via the atrioventricular node and the His-Purkinje system would show distinctly different patterns of ventricular activation (Fig. $1 D$ ). An example of plaque recording in another patient with two accessory pathways is shown in Fig. 2.

Ventricular insertion site of an accessory pathway was defined as the site of accessory pathway potential or, in its absence, the location of the earliest ventricular epicardial breakthrough during preexcitation (Fig. $1 \mathrm{~A}$ ). Atrial insertion site of an accessory pathway was the earliest atrial epicardial breakthrough during ventricular pacing (Fig. $1 B$ ) or the atrial site closest to the ventricular insertion site. Activations a this site represent atrial inputs into the accessory pathway during atrial fibrillation.

A spatially uniform atrial wavefront is one which consists of a single large wavefront (Fig. $1 C$ ), in contrast to fractionated and curved wavefronts which approach the accessory pathway from multiple foci and with sharp curvatures (Fig. 1 D). These are spatial characteristics of atrial wavefronts. Temporal characteristics were also analyzed to account for the "prematurity" of atrial wavefronts relative to the accessory pathway refractoriness. Because accessory pathway potentials were not consistently recorded, the exact time of accessory pathway activation could not be determined. However, it could be approximated either by the time of atrial wavefront arrival at the accessory pathway atrial insertion site, or by the time of earliest ventricular activation at the accessory pathway ventricular insertion site. Thus, two new variables were defined (Fig. 3). A-A interval was the interval from the arrival time of the previous atrial wavefront that depolarized the accessory pathway to the arrival time of the index atrial wavefront. V-A interval was the interval from the time of previous local ventricular activation to the arrival time of the index atrial wavefront. Time intervals were taken at the atrial and ventricular accessory pathway insertion sites, respectively, to minimize time delay due to intra-atrial or intra-ventricular conduction. Although both variables were indices of the "prematurity" of the atrial wavefronts, the two differed by the conduction time of the accessory pathway. Note that A-A interval could not be determined for atrial wavefronts following nonpreexcited QRS complexes (Fig. 3, atrial complexes 7 to 9 ), since the atrial wavefront immediately preceding the QRS bears no relationship to accessory pathway conduction (6th atrial activation). In contrast, V-A intervals were not limited by this factor, assuming that retrograde conduction into the accessory pathway occurred with non-preexcited complexes.

Statistical analysis. Statistical analyses were performed using STATISTICA software packages (StatSoft, Inc., Tulsa, OK). Fisher's exact test was used to compare proportion of fractionated atrial wavefronts that resulted in accessory pathway conduction versus that of uniform atrial wavefronts. The null hypothesis was rejected if the $P$ value was $<0.05$.

\section{Results}

The clinical characteristics of the eight patients are listed in Table I. All patients had symptomatic and drug refractory preexcitation syndrome and had $1(n=6)$ or $2(n=2)$ accessory pathways. All accessory pathways conducted bidirectionally. 10 episodes of QRS transition from consecutively preexcited morphology to nonpreexcited morphology were identified in these patients.

The relation between atrial wavefront characteristics and accessory pathway conduction. In 8 of 10 episodes of QRS transition from consecutively preexcited to non-preexcited complexes, there were significant changes in atrial wavefront characteristics before loss of accessory pathway conduction. Uniform large atrial wavefronts were associated with atrioventricular conduction through the accessory pathway. Immediately prior to QRS transition, however, four general types of changes in atrial wavefronts were noted: $(a)$ premature invasion by secondary wavefronts creating local conduction block with subsequent failure of impulse propagation through the accessory pathway $(n=5)$; $(b)$ wavefront collision leading to curvature of wavefront propagation centering around an area of conduction block, with subsequent loss of accessory pathway conduction $(n=2) ;(c)$ uniform large atrial wavefronts undergoing a transition into multiple smaller wavefronts followed by loss of accessory pathway conduction $(n=1)$; and $(d)$ uniform large atrial wavefronts appearing to "dissociate" from the ventricle and "marching" into the refractory period of the accessory pathway $(n=2)$.

Wavefront invasion. Fig. 4 shows an example of QRS transition in patient 7 , who has a single left lateral accessory pathway. Uniform large atrial wavefronts from the right upper corner of 
A

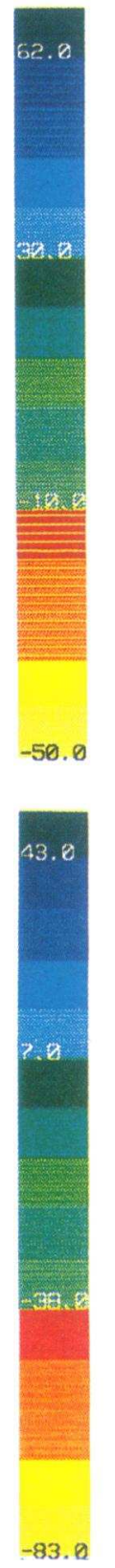

B
Sinus Rhythm

(with Ventricular Preexcitation)

\section{Atrium}

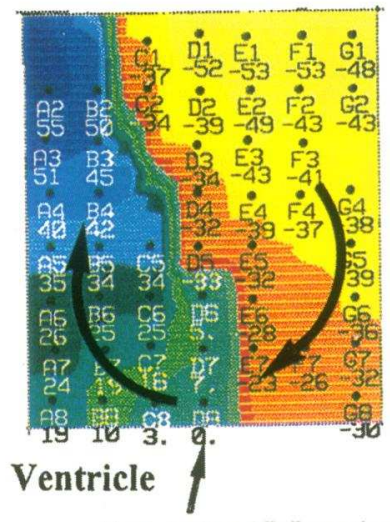

Ventricular AP Insertion Site

C

(with Ventricular Preexcitation)

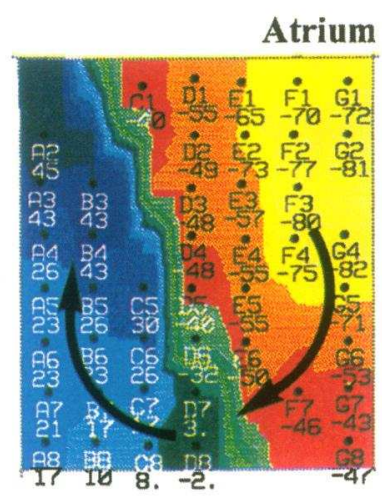

Ventricle
Atrial Fibrillation

\section{Ventricular Pacing \\ (with Atrial Preexcitation)}

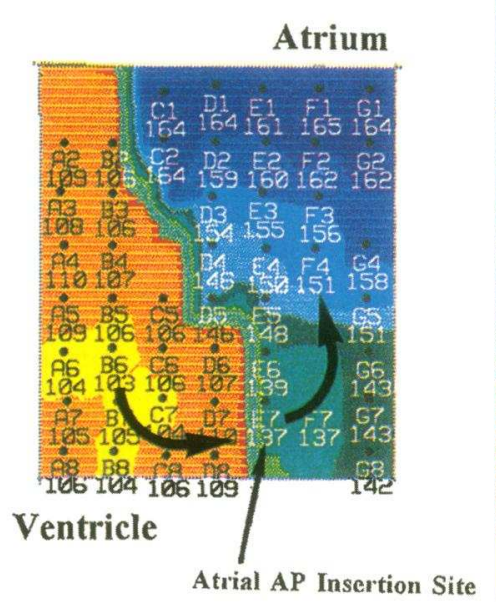

D
Atrial Fibrillation

(No Preexcitation)

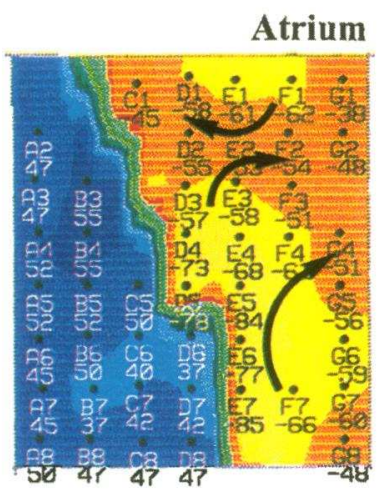

Ventricle
Figure 1. An example of activation mapping with the plaque electrode array in patient 7 . Isochronal maps are drawn to maximize temporal differences in color. Time 0 is set to the onset of surface QRS. Yellow color represents the earliest activation and deep blue the latest (see vertical bars). During preexcited beats in sinus rhythm $(A)$ or atrial fibrillation $(C)$, the earliest ventricular epicardial activation was at electrode $\mathrm{D} 8$, representing the ventricular insertion site of the accessory pathway. Activation then spread centrifugally from this point. During ventricular pacing $(B)$, the earliest retrograde atrial activation was at electrode E7, representing the atrial insertion site of the accessory pathway. During atrial fibrillation, the local activation pattern with ventricular preexcitation $(C)$ is distinguished from a non-preexcited beat activated from the atrioventricular node $(D)$. The latter pattern of activation was relatively homogeneous, consistent with activation from the endocardium to the epicardium through the Purkinje fibers. $A P$, accessory atrioventricu-54.0 lar pathway. the recording plaque activated the accessory pathway with a stable 2:1 ratio, leading to ventricular preexcitation (panels 2, 4,6 , and 8 ). Atrial wavefronts 3,5 , and 7 , on the other hand, did not activate the accessory pathway due to accessory pathway refractoriness, even though they were uniform large wavefronts (panels 5 and 7). The 9th atrial wavefront, however, originated from a different focus (the right edge of the recording plaque). Because this new wavefront invaded the mapped area prematurely $(\sim 30 \mathrm{~ms}$ after previous accessory pathway activation, see panel 9), it encountered refractory tissues near electrode G6/G7, resulting in conduction block. The wavefront then propagated around this area of conduction block (note crowding of isochronal lines near electrodes $\mathrm{G6/G7}$ ) and failed to activate the accessory pathway. A similar wavefront (No. 10) entered the area, encountering a larger area of conduction block, and failed to activate the accessory pathway, despite its arrival nearly $300 \mathrm{~ms}$ after the previous accessory pathway activation (see accessory pathway atrial insertion site, electrode E7, panel 10). Thus, in this episode, loss of accessory pathway conduction was preceded by premature invasion by a secondary atrial wavefront, resulting in local conduction block and change of wavefront direction. Finally, return of a large atrial wavefront from the right upper corner (No. 11 ) resulted once again in accessory pathway activation. However, enough time has elapsed since the previous activation, allowing time for atrioventricular nodal conduction and resultant fusion beat (QRS No. 5). Subsequent uniform atrial wavefronts resumed regular accessory pathway activation (atrial wavefronts not shown).

Another episode of QRS transition of the same patient is shown in Fig. 5. The first three uniform atrial wavefronts from the right upper corner of the recording plaque activated the accessory pathway with a $2: 1$ ratio (only the 3rd wavefront is 
A

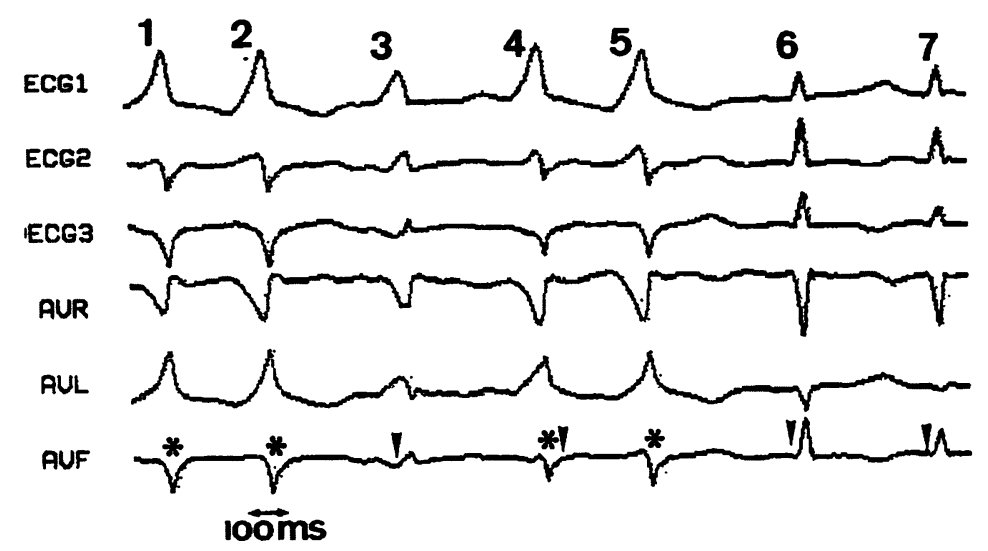

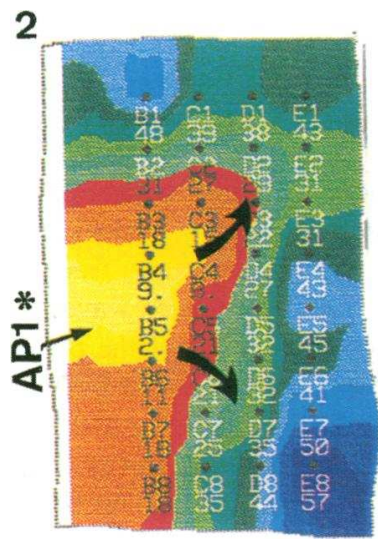

5

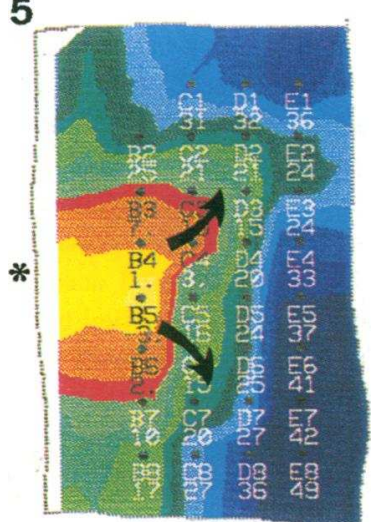

3

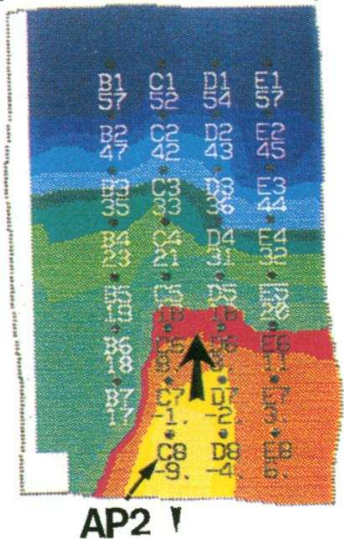

6

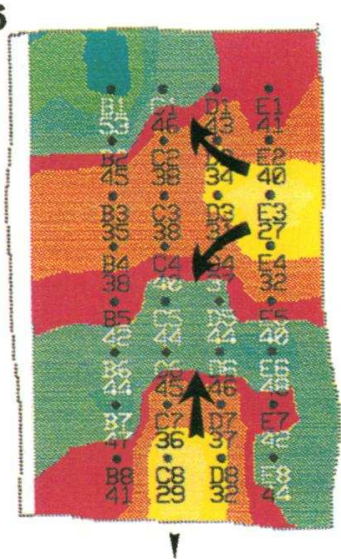

4

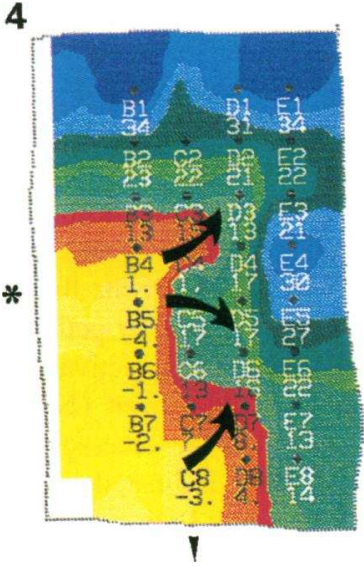

7

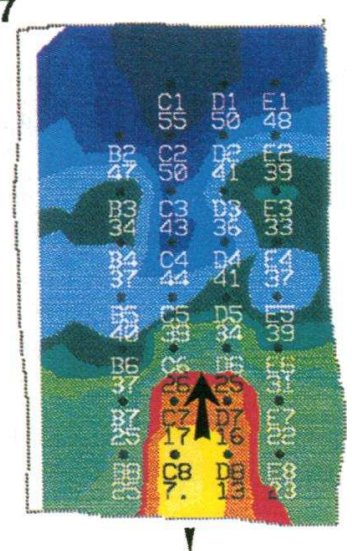

Figure 2. Plaque recording in patient 1 with a left posterior and a right posteroseptal accessory pathway. Only ventricular activations are drawn for clarity (time 0 set at the onset of surface QRS). $A$ shows surface ECG of a run of atrial fibrillation with 7 QRS complexes. QRS $1,2,4$ and 5 are preexcited complexes from activation of accessory pathway No. 1 (right posteroseptal, marked with an asterisk) while QRS 3 is a preexcited complex from activation of accessory pathway No. 2 (left posterior, marked with an inverse arrowhead). In panels 2 and 5 , ventricular preexcitation is seen beginning near electrodes B4/B5 (accessory pathway ventricular insertion site). Activation of accessory pathway No. 2 shows a distinctly different pattern (panel 3). QRS No. 4 (panel 4) shows a fusion beat from both accessory pathways. QRS 6 and 7 are fusion beats from accessory pathway No. 2 and atrioventricular node, with different degree of ventricular preexcitation. Note that antegrade accessory pathway activation may occur after the onset of QRS, as long as activation from the atrioventricular node has not reached the accessory pathway (panel 6). AP, accessory atrioventricular pathway.

shown). The 4th wavefront encountered an area of conduction block near electrodes G5/G6 and propagated around it. A similar wavefront (No. 5) also failed to activate the accessory pathway. Later, a uniform large atrial wavefront (No. 6) from the right lower corner invaded the area and activated the accessory pathway (QRS No. 3). A new wavefront (No. 7) invaded prematurely ( $105 \mathrm{~ms}$ after accessory pathway activation) from the right of the recording plaque near electrodes G4/G5, and again 


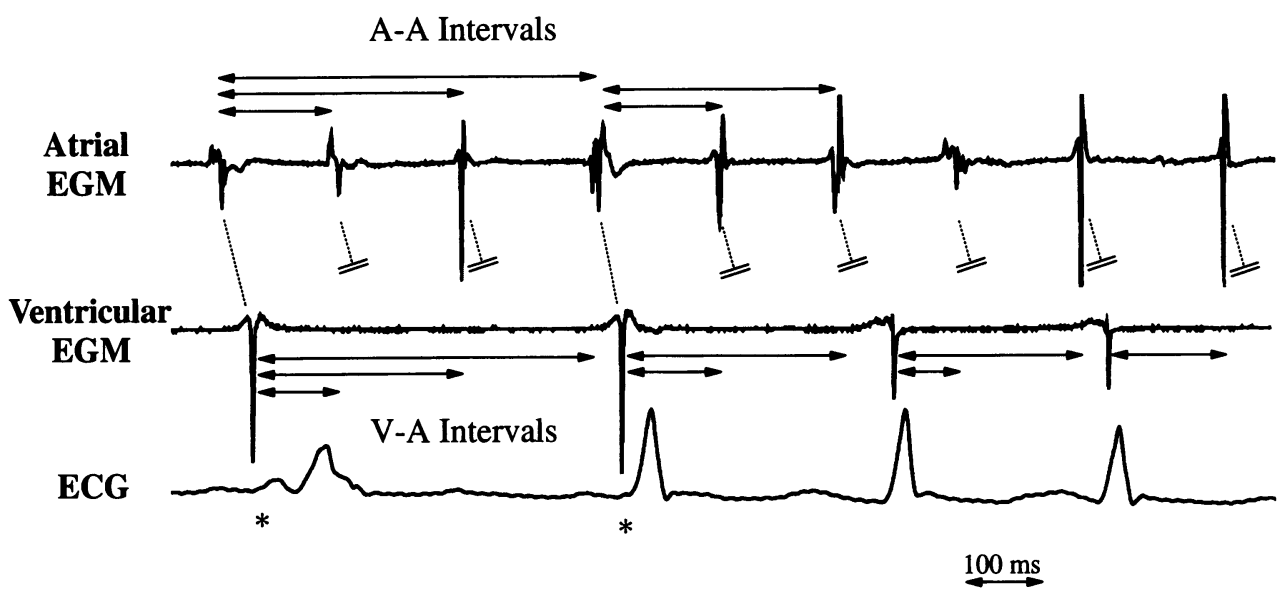

Figure 3. Definition of A-A and V-A intervals. The top and the middle rows consist of local bipolar electrograms recorded from the atrial and ventricular insertion sites of an accessory pathway, respectively. The bottom row is surface electrocardiogram. Only the first and fourth atrial wavefronts lead to accessory pathway conduction (preexcited and fusion QRS complexes are marked by an asterisk). Note changes in local ventricular activations with (first and second QRS complexes) and without (third and fourth QRS complexes) accessory pathway conduction. A-A interval was the

time elapsed since the previous atrial wavefront that depolarized the accessory pathway, whereas V-A interval was that since the previous local ventricular activation. Both intervals "reset" after each QRS complex, but A-A intervals could not be determined following non-preexcited QRS complexes. See text for details. EGM, electrogram: $E C G$, electrocardiogram.

in the next cycle (No. 8). These premature wavefronts fractionated into several smaller wavelets and propagated in different directions at sharp curvatures, failing to activate the accessory pathway, despite their arrival at the accessory pathway nearly $300 \mathrm{~ms}$ after the previous accessory pathway activation (panels 7 and 8). The next uniform large wavefront (No. 9) from the right upper corner again activated the accessory pathway. Wavefront No. 10 appeared to have originated endocardially (5) and spread in different directions, failing to activate the accessory pathway. Wavefront No. 12 showed retrograde conduction of ventricular activation through the accessory pathway into the atrium. This retrograde penetration may have prolonged accessory pathway refractoriness. All subsequent atrial wavefronts (e.g., No. 13) failed to activate the accessory pathway despite the fact that uniform wavefronts arrived outside of the apparent accessory pathway refractory period.

Wavefronts collision and curvature. Collision of atrial wavefronts during QRS transition was seen in a patient (No. 1) with 2 accessory pathways (left posterior and right posteroseptal), shown in Fig. 6. Two large atrial wavefronts were recorded in this episode. An early atrial wavefront from the left

Table I. Clinical Characteristics

\begin{tabular}{ccccccc}
\hline Patient & Age & $\begin{array}{c}\text { Accessory } \\
\text { pathway }\end{array}$ & $\begin{array}{c}\text { Organic } \\
\text { disease }\end{array}$ & Symptom & $\begin{array}{c}\text { H/O } \\
\text { SVT }\end{array}$ & $\begin{array}{c}\text { H/O } \\
\text { AF }\end{array}$ \\
\hline 1 & 18 & LP, RAPS & none & palpitation & YES & NO \\
2 & 55 & LL & CAD & palpitation & YES & YES \\
3 & 23 & LL & none & palpitation & YES & NO \\
4 & 42 & LL & none & palpitation & YES & NO \\
5 & 45 & LL & none & palpitation & YES & NO \\
6 & 30 & LP & none & palpitation & YES & NO \\
7 & 31 & LL & none & palpitation & NO & YES \\
8 & 20 & LP, RPS & none & syncope & NO & NO \\
& $32 \pm 12$ & & & & & \\
& & & & & &
\end{tabular}

$A F$, atrial fibrillation; $A P$, accessory atrio-ventricular pathway; $C A D$, coronary artery disease; $H / O$, history of; $L L$, left lateral; $L P$, left posterior; $R P S$, right posteroseptal; $S V T$, supraventricular tachycardia. upper corner of the recording plaque near electrodes A1/A2 (designated wavefront $\mathrm{A}$ ) encountered conduction block near electrodes A3/A4. A second large uniform wavefront from the lower left corner near electrodes A5/A6 (designated wavefront B) depolarized the area in the left lower corner of the plaque and activated accessory pathway No. 1 and accessory pathway No. 2 (electrodes C4 and C8, respectively). Thus, the first QRS was the result of fusion from both accessory pathways. The next group of wavefronts (No. 2) arrived within the refractory period of both accessory pathways and failed to conduct. The next group of wavefronts (panel 3) resulted from fusion of wavefronts A and B, activating accessory pathway No. 1. The fusion wavefront encountered conduction block near electrodes A6/A7 but proceeded to activate a narrow strand of atrial tissue near accessory pathway No. 2 (electrodes B6 to B8). Ventricular depolarization from accessory pathway No. 1 spread toward accessory pathway No. 2 (electrode C8), preventing antegrade activation of accessory pathway No. 2 by the fusion wavefront. The next wavefront $B$, which previously activated accessory pathway No. 2, arrived late (shown in blue) and failed to activate accessory pathway No. 2 . The next two groups of wavefronts (Nos. 4 and 5) consisted of alternating invasion of wavefronts A and B into the area near accessory pathway No. 1, preventing each other from activating accessory pathway No. 1. Despite the late arrival of a wavefront B (over $400 \mathrm{~ms}$ after the previous accessory pathway No. 1 activation, marked + in panel $A$ ), it failed to activate accessory pathway No. 1. However, this wavefront spread toward the lower corner and activated accessory pathway No. 2. Enough time has elapsed to allow atrioventricular nodal conduction and fusion complex (QRS No. 3). Thus, QRS transition in this patient was preceded by multiple wavefronts colliding with and inhibiting each other.

Fig. 7 is an example of wavefront collision and curvature during QRS transition in patient 5. A uniform large wavefront propagated from left to right across the recording plaque, activating the accessory pathway and resulted in a preexcited QRS (No. 1). Atrial double potentials were seen starting with the second wavefront, representing slow conduction and collision with another wavefront coming from the right of the plaque (panel 2). The next wavefront (No. 3) arrived and activated 


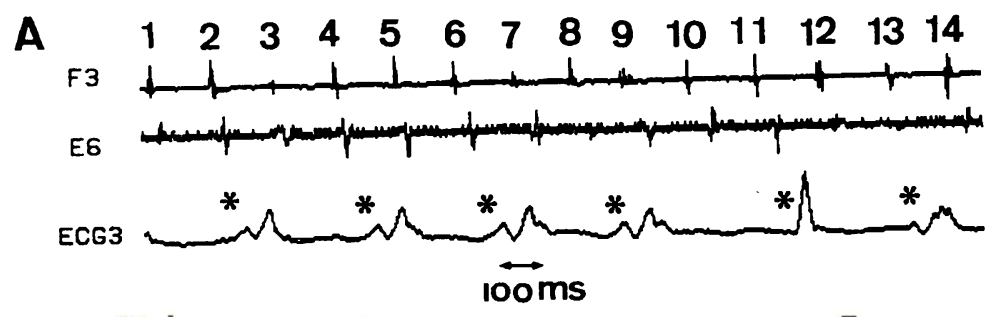

2

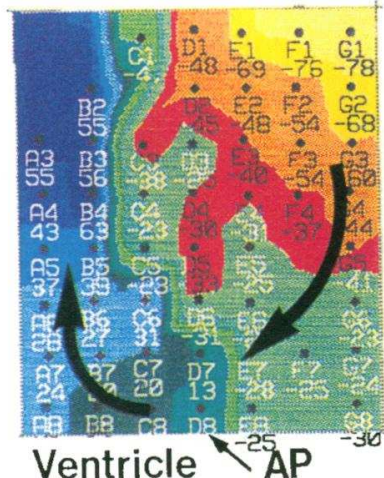

6

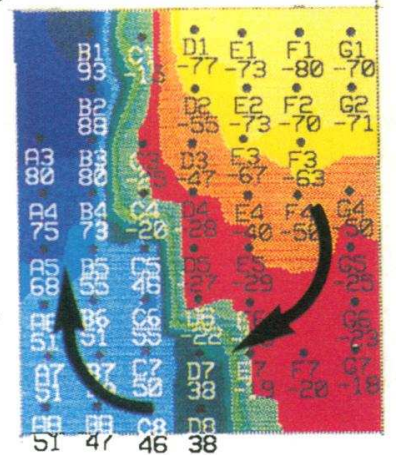

9

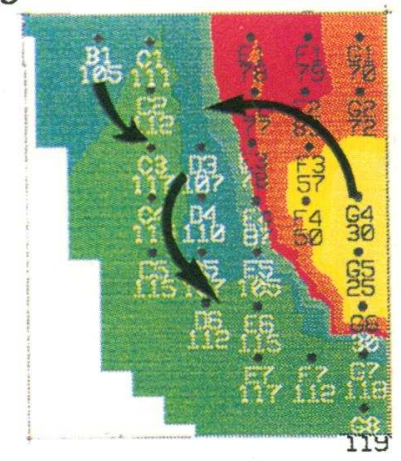

4

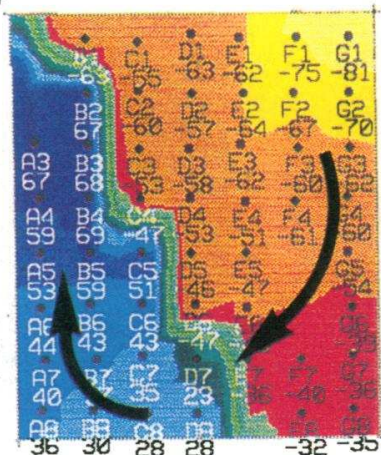

7

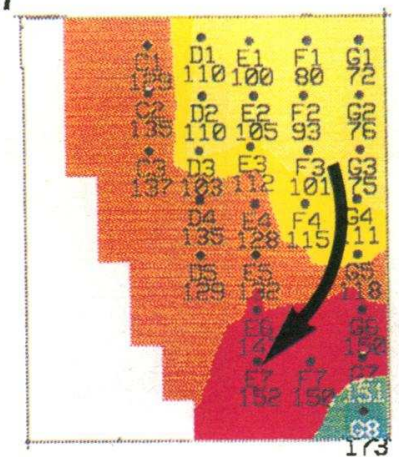

10

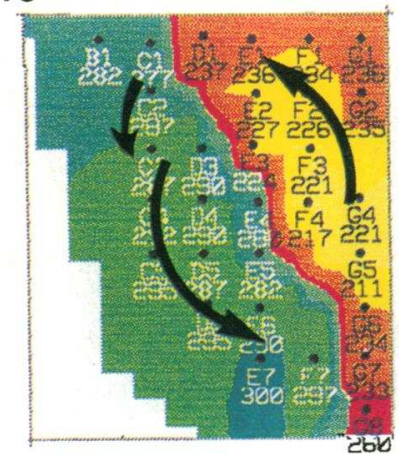

5

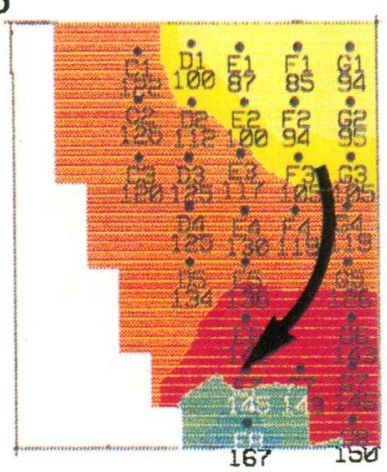

8

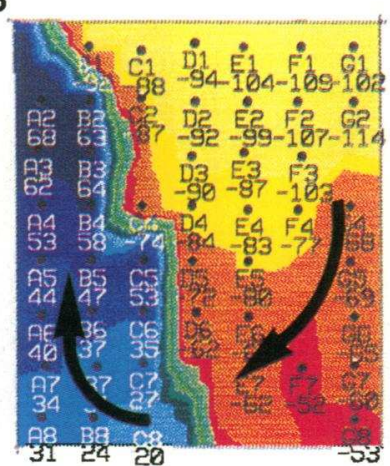

11

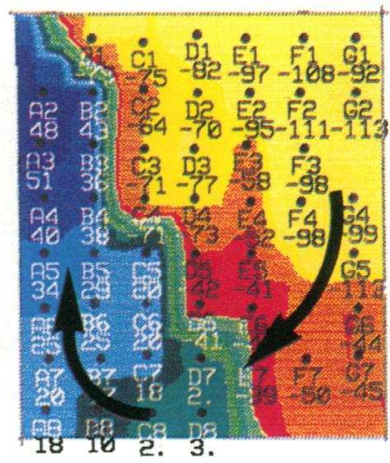

Figure 4. An episode of transition of QRS morphology during atrial fibrillation in patient 7 (left lateral accessory pathway). A shows the atrial electrogram recorded at electrodes F3 and E6 and surface ECG lead III (ventricular preexcitation is marked by an asterisk). Time 0 is set to the onset of QRS. Yellow color represent the earliest and deep blue the latest activation. Uniform large atrial wavefronts from the right upper corner of the recording plaque lead to accessory pathway activation. Invasion by a secondary wavefront from the right (panels 9 and 10 ) resulted in loss of accessory pathway conduction and QRS transition. For details, see text. AP, accessory atrioventricular pathway.

the accessory pathway before colliding with another wavefront from the right. Starting with atrial wavefront No. 4, curvature of wavefront propagation was noted at the area of previous wavefront collision, around a center of conduction block (electrodes E1/F1). However, the next large and uniform wavefront (No. 5) arrived from the left and activated the accessory path- way. A new wavefront (No. 6) coming from the left upper corner of the plaque encountered conduction block at E1/F1 junction and propagated around it with a sharp curvature. All subsequent atrial wavefronts conducted with similar curvature around a center of slow conduction and conduction block, with failure to activate the accessory pathway and loss of preexcita- 


\section{A}

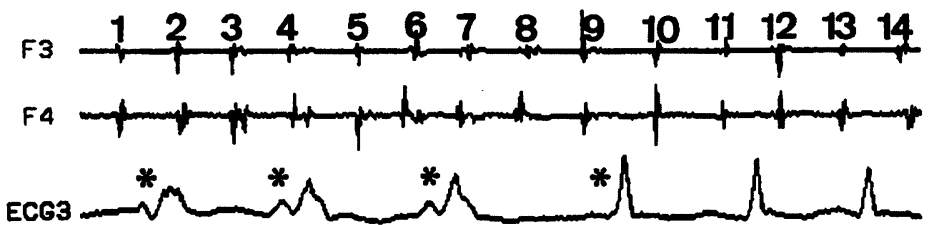

$\stackrel{\leftrightarrow}{100 \mathrm{~ms}}$

3

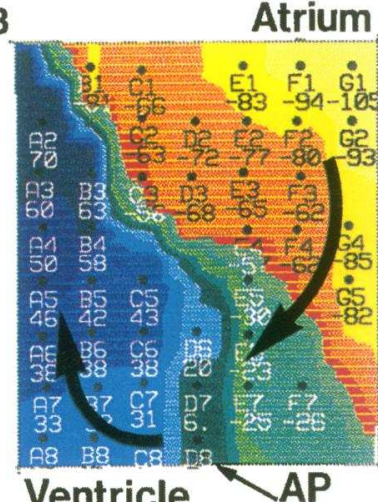

7

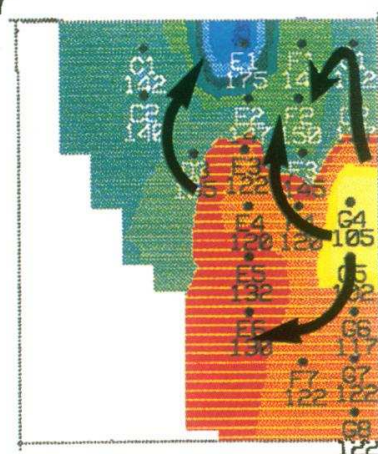

10

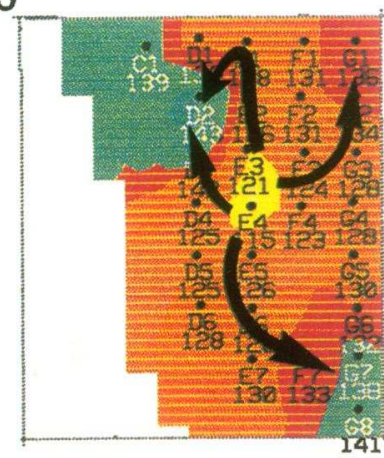

4

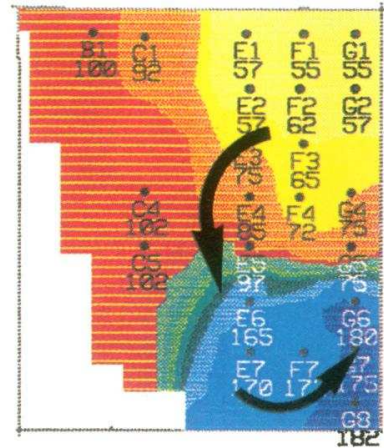

8

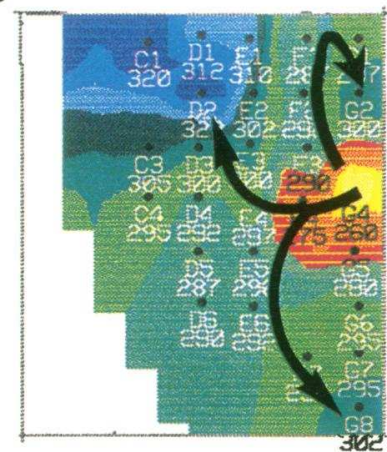

12

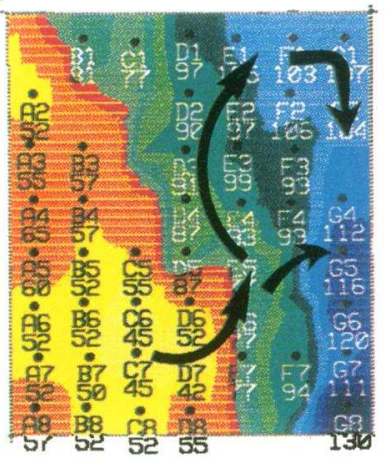

6

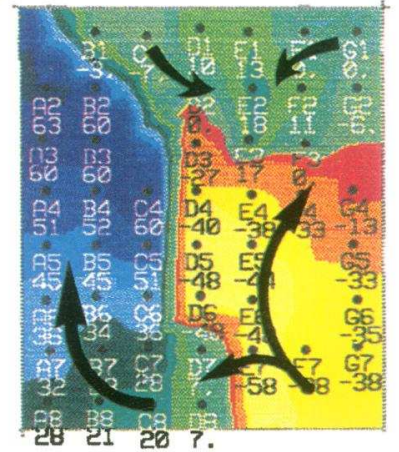

9

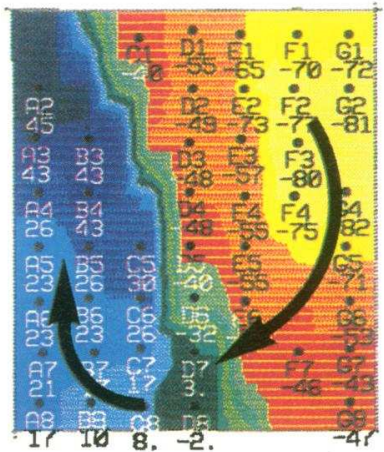

13

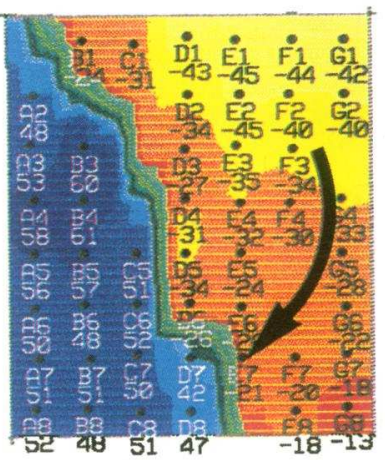

Figure 5. Another episode of transition of QRS morphology in patient 7. A shows the atrial electrogram recorded at electrodes F3 and F4 and surface ECG lead III (ventricular preexcitation is marked by an asterisk). QRS transition is characterized by invasion by a new wavefront (panel 7 and 8 ) which split into multiple smaller wavefronts with failure to activate the accessory pathway. After loss of preexcitation, retrograde invasion of the accessory pathway was noted in panel 12. For details, see text.

tion. Note also differences in ventricular activation (E8) before and after QRS transition. Atrial double potentials in this patient represent areas of wavefront collision and slow conduction, which are associated with wavefront curvature and failure of accessory pathway conduction.

Wavefront breakup. A third type of QRS transition was associated with highly fractionated atrial electrograms near the atrial accessory pathway insertion site. Fig. 8 is an example of QRS transition in patient 2 (a single left lateral accessory pathway), in whom several episodes of QRS transitions were noted during $8 \mathrm{~s}$ of recording. Only a representative isochronal map of a preexcited beat is shown. In a typical episode, electrodes adjacent to the accessory pathway atrial insertion site (A1 and A2) recorded fractionated atrial electrograms during QRS transition from preexcited to nonpreexcited complexes (note changes in ventricular activation in electrode A6). Start- 
$\mathbf{A}$

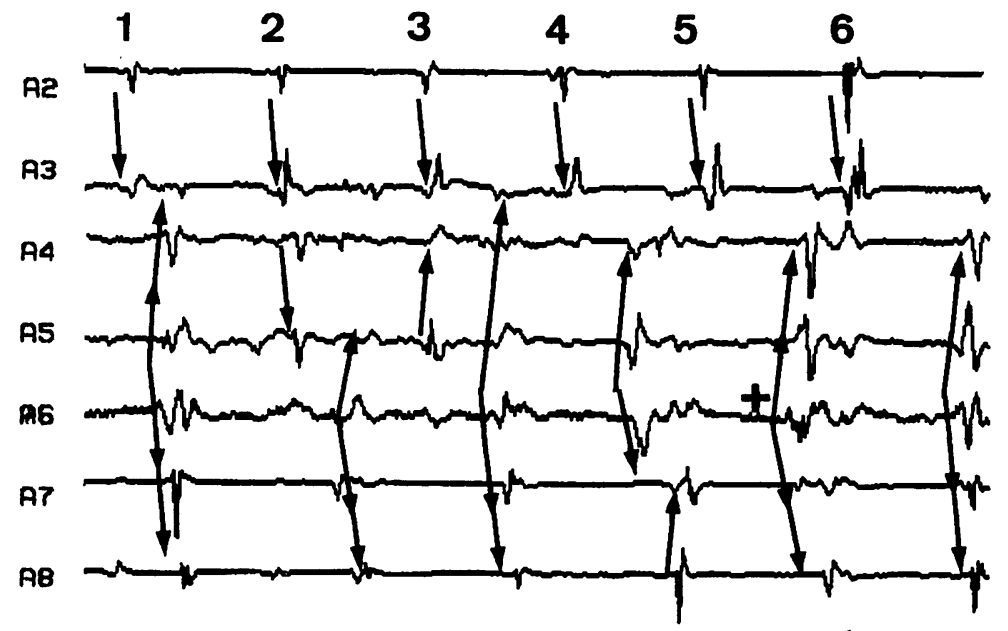

AUF

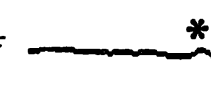

B

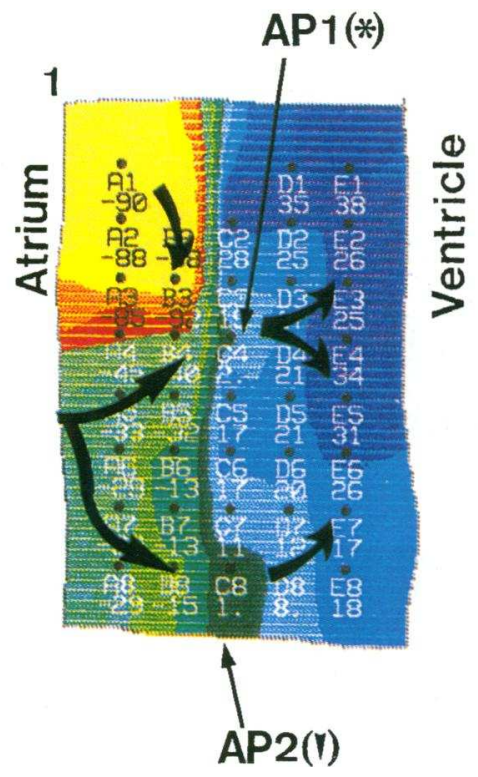

10Oms

\section{3}

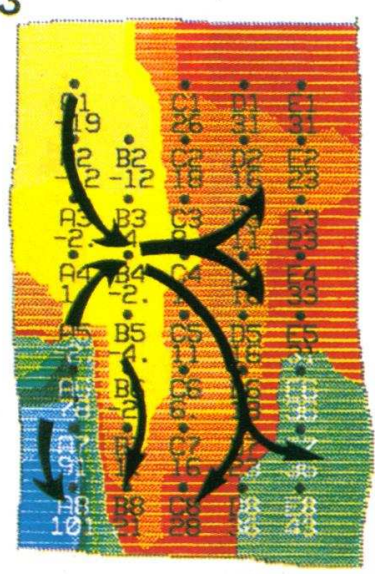

Figure 6. An example of QRS transition in patient 1 ( 2 accessory pathways, see also Fig. 2). A shows atrial electrograms (A2 to A8) and surface ECG lead AVF. The accessory pathways are located near electrodes C4 and C8. Only two representative isochronal maps (atrial complexes 1 and 3 ) are shown in $B$. Ventricular preexcitation is marked by an asterisk for accessory pathway No. 1 and by an inverse arrowhead for accessory pathway No. 2. Time 0 is set to the onset of QRS. In this run of atrial fibrillation, fusion of both accessory pathways (QRS No. 1), summation (atrial complex No. 3) and inhibition (atrial complexes Nos. 4 to 6) of atrial wavefronts, and retrograde concealment of accessory pathway conduction (QRS No. 2) can be observed. See text for details. $A P$, accessory atrioventricular pathway. ing with wavefront No. 5 (arrow), increasing degree of disorganization and fractionation of atrial electrograms and reduction of their amplitudes were noted until the accessory pathway failed to conduct by the 4th QRS complex. With return of organized atrial activities, accessory pathway resumed conduction (not shown). Changes in atrial activations were seen first in electrode $\mathrm{C} 1$, followed by $\mathrm{B} 2, \mathrm{~A} 2$, and A1. Loss of accessory pathway conduction occurred as the disturbance approached the accessory pathway insertion site at A2. This pattern of wavefront fractionation and loss of accessory pathway conduction recurred several times during the $8 \mathrm{~s}$ of recording.

Role of accessory pathway refractory period. A fourth type of QRS transition is exemplified in Fig. 9, patient 3 (a single left lateral accessory pathway). A representative isochronal map of a preexcited beat is shown on the right. A uniform large atrial wavefront approached the accessory pathway from the left upper corner of the recording plaque and propagated toward the left lower corner, activating the accessory pathway. There were no significant changes in the characteristics of atrial wavefronts or the atrial coupling interval during the period of observation. However, as the wavefronts arrived at different time, different degrees of ventricular fusion were noted. When wavefronts arrived within the accessory pathway refractoriness (e.g., wavefronts Nos. 1, 3, 5, 6, 7, 8, and 10), no accessory pathway activation occurred. When they arrived outside of the accessory pathway refractoriness (wavefronts Nos. 2, 4, and 9), accessory pathway activation occurred. Note also changes in local ventricular bipolar electrogram (electrode F8) with ventricular preexcitation.

Temporal characteristics of atrial wavefronts and accessory pathway conduction. In most episodes, spatial geometry of atrial wavefronts appears to be the main determinant for accessory pathway conduction during QRS transition. However, we hypothesized that in addition to spatial geometry, temporal characteristics may also affect accessory pathway conduction. Sourcesink hypothesis predicts that premature atrial wavefronts with 


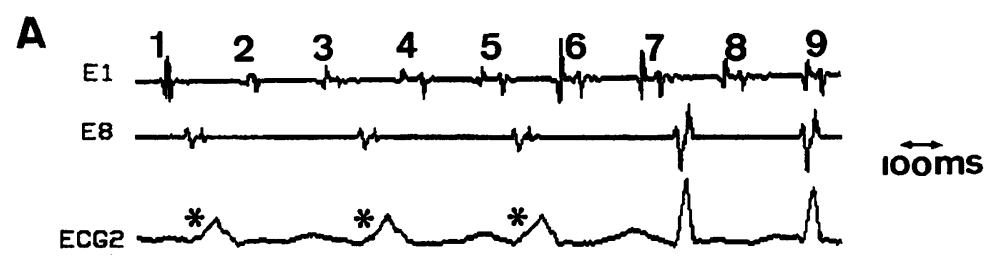

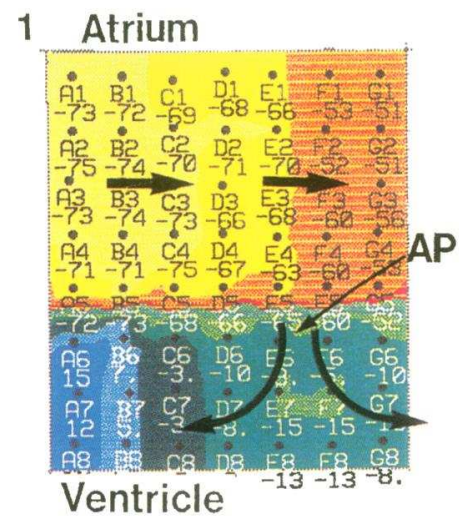

3

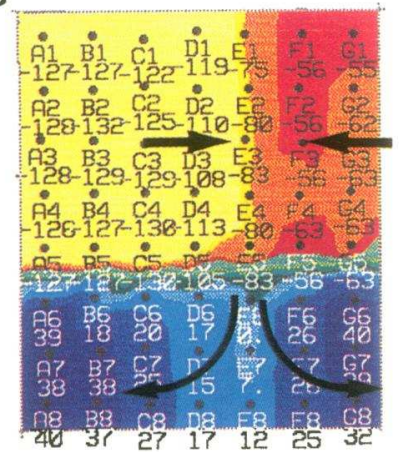

6

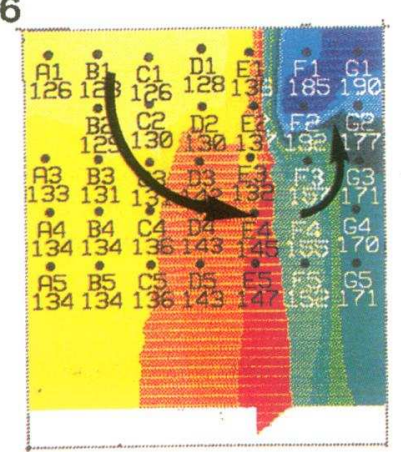

4

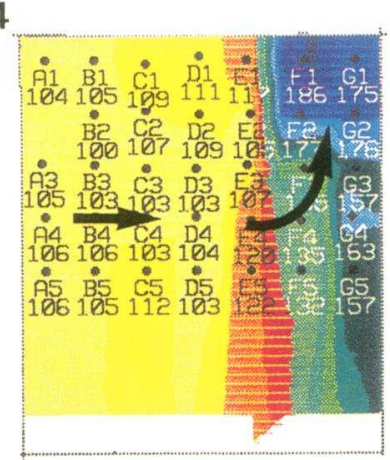

7

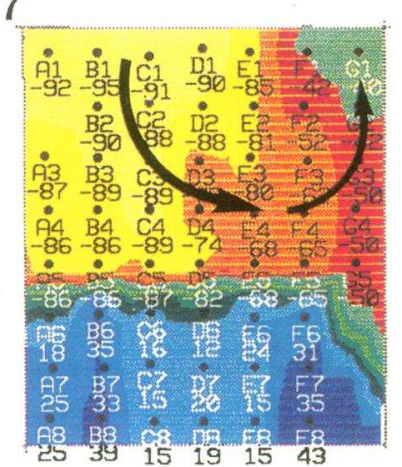

5

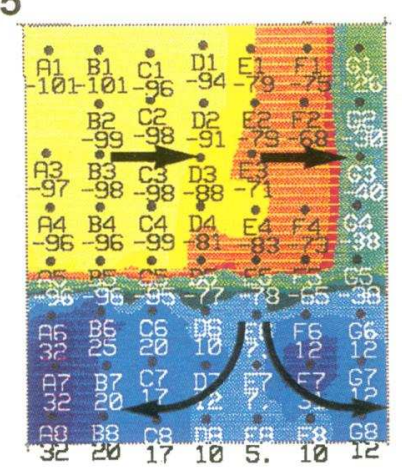

8

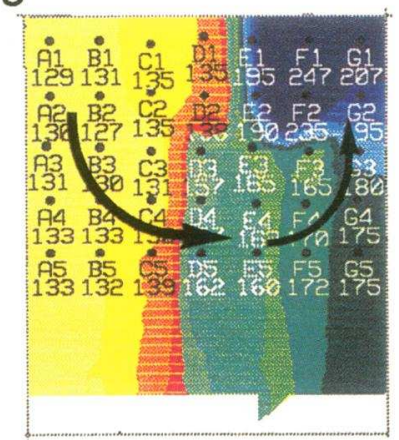

Figure 7. QRS transition in patient 5 (left lateral accessory pathway). A shows atrial (electrode E1) and ventricular (electrode E8) activations, along with surface ECG lead II. Preexcited beats are marked by an asterisk. Isochronal maps are drawn to maximize temporal differences in color, with time 0 set to the onset of surface QRS. In general, uniform large atrial wavefronts arriving at the accessory pathway resulted in activation of the accessory pathways. Starting with the second atrial wavefront, double atrial potentials are seen, representing areas of slow conduction and wavefront collision (panel 2). After wavefront collision, curvature of wavefront propagation is noted starting with wavefront No. 4, with subsequent loss of preexcitation by wavefront No. 7. Note changes in ventricular activation (E8) during the transition. For details, see text. AP, accessory atrioventricular pathway.

more rapid arrival would be less likely to conduct than one with a slower rate $(2,3)$. To evaluate this, both spatial and temporal characteristics of each atrial wavefront were analyzed. Fig. 10 shows a comparison of fractionated versus uniform wavefronts plotted against their respective A-A interval (panel $A$ ) and
V-A interval (panel $B$ ) in five patients. Two patients did not exhibit wavefront changes during QRS transition (see Fig. 9) and were not included in this analysis. In another patient, the plaque array did not record atrial wavefronts adequately to determine their spatial characteristics. 

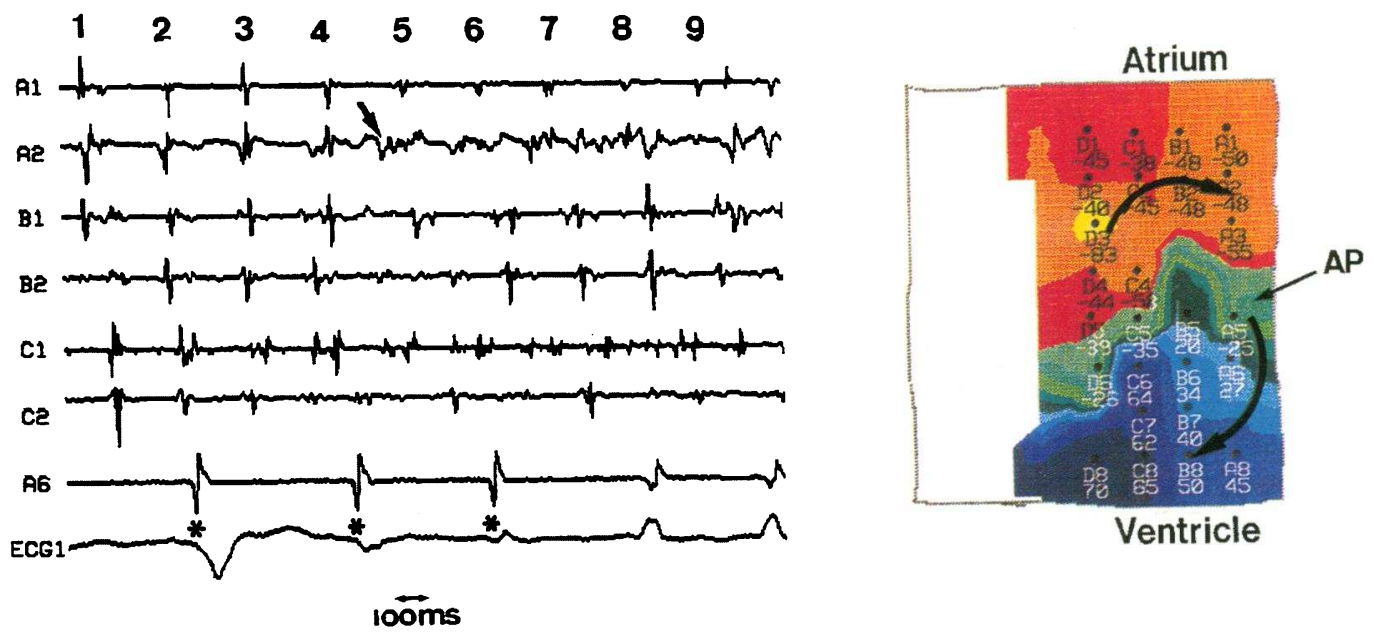

Figure 8. QRS transition in patient 2 with a single left lateral accessory pathway. Activations and ECG are shown on the left. A representative isochronal map of a preexcited beat is shown on the right. Time 0 is set at the onset of surface QRS. Preexcited complexes are marked with an asterisk. In this patient, double atrial potentials and fractionated atrial electrograms were recorded starting with the 2nd atrial complex in electrode C1. Atrial electrograms at the accessory pathway atrial insertion site (electrode A2) became increasingly disorganized starting with atrial complex No. 5 (arrow), followed shortly by loss of accessory pathway conduction. AP, accessory atrioventricular pathway.

In Fig. $10 A$ (patient 1 ), 1 of 18 fractionated wavefronts lead to accessory pathway conduction, compared to 3 of 7 uniform wavefronts $(P=0.05)$. Similar results were noted for two other patients. However, for patients 5 and 7 , the number of atrial wavefronts analyzed were too small to reach statistical significance, but a trend was present. Note that at intervals above the horizontal bars (the shortest A-A interval that resulted in accessory pathway conduction), uniform wavefronts are more likely to conduct than fractionated ones (pooled data: 3 of 15 fractionated vs. 42 of 47 uniform wavefronts, $P<0.001$ ). This suggests that at intervals beyond the accessory pathway refractoriness, success of conduction depends on spatial characteristics of atrial wavefronts. Similar conclusions were reached analyzing V-A intervals. In patient 1, Fig. $10 B, 2$ of 57 fractionated wavefronts lead to accessory pathway conduction, compared to 5 of 19 uniform wavefronts $(P=0.009)$. The differ- ence was significant for two other patients. Note again that beyond the accessory pathway refractoriness (horizontal bars), many fractionated wavefronts failed to preexcite the ventricle, whereas most uniform wavefronts did (pooled data: 5 of 34 fractionated vs. 49 of 66 uniform wavefronts, $P<0.001$ ).

\section{Discussion}

Atrial wavefront characteristics and accessory pathway conduction. In this study, we found that both spatial and temporal characteristics of atrial wavefronts during atrial fibrillation are important determinants of accessory pathway conduction. By analyzing atrial activation patterns during QRS transition from consecutively preexcited to nonpreexcited complexes, one can readily demonstrate factors contributing to loss of accessory pathway conduction during atrial fibrillation. In all patients,
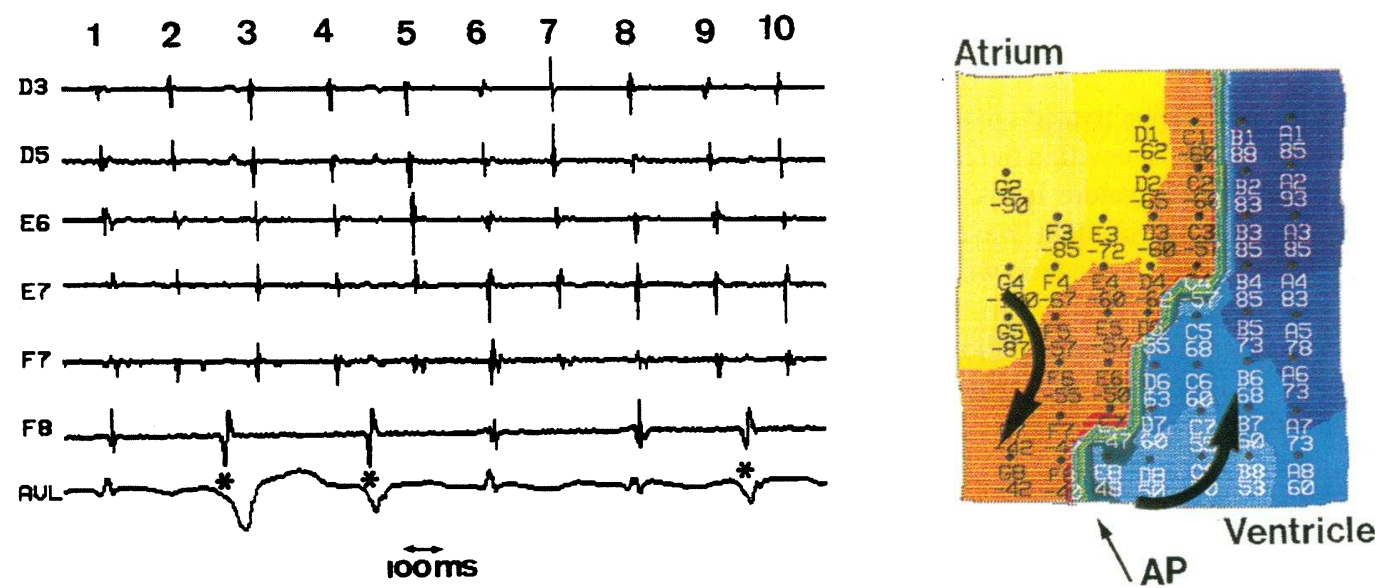

Figure 9. QRS transition in patient 3 with a single left lateral accessory pathway. Activations and ECG are shown on the left. A representative isochronal map of a preexcited beat is shown on the right. Time 0 is set at the onset of surface QRS. Preexcited complexes are marked with an asterisk. There was no significant change in atrial activations with QRS transition. The degree of preexcitation in the fusion beats is a function of timing of arrival of atrial wavefronts. Note also changes in ventricular activations with preexcitation (electrode F8). AP, accessory atrioventricular pathway. 

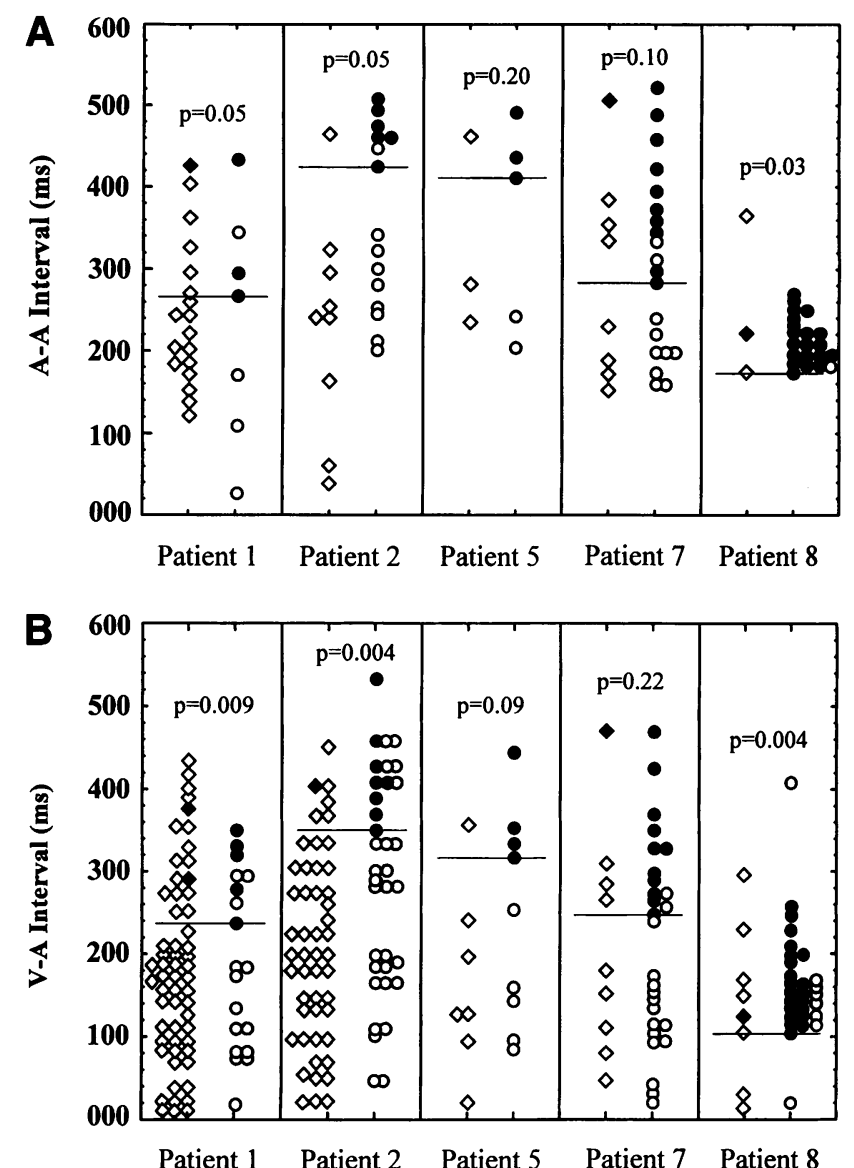

Figure 10. Comparison of uniform and fractionated atrial wavefronts in 5 patients. Spatial characteristics of each atrial wavefront are plotted against the respective A-A $(A)$ and $\mathrm{V}-\mathrm{A}$ intervals $(B)$. Diamonds represent fractionated atrial wavefronts that did (filled diamonds) and did not (open diamonds) activate the accessory pathway. Similarly, circles represent uniform atrial wavefronts that did (filled circles) and did not (open circles) activate the accessory pathway. Horizontal bars represent the shortest A-A or V-A intervals that lead to accessory pathway conduction for each patient. Below these bars, accessory pathway conduction is mainly governed by its refractoriness. Above them, wavefront spatial characteristics become important. For details, see text. $A P$, accessory atrioventricular pathway.

uniform large atrial wavefronts consistently activated the accessory pathway, provided that they did not arrive within the refractory period of the accessory pathway. Premature invasion by impulses from a different direction with resultant wavefront collision, wavefront curvature, and wavefront breakup were associated with loss of accessory pathway conduction. These findings support the hypothesis that the source-sink relationship is an important factor in determining impulse propagation through the accessory pathway during atrial fibrillation.

Source-sink hypothesis. In patients with the preexcitation syndrome, the atrium serves as the source for a smaller sink, the accessory pathway, which in turn acts as a source for a larger sink, the ventricular myocardium. During atrial fibrillation, atrial activation wavefronts with varying amount of electrotonic current arrive at the accessory pathway at irregular intervals. Experimental studies by de la Fuente (2) and Cabo (3) suggest that variation in the quality of atrial input into the accessory pathway can affect its output at the ventricular inser- tion site, which ultimately determines ventricular preexcitation. As shown by de la Fuente et al. (2) in an in vitro model of the preexcitation syndrome, when impulses from one end of a narrow isthmus propagating across the isthmus failed to excite the other end (e.g., with rapid pacing or hyperkalemia), conduction block consistently occurred just distal to the junction where the small isthmus inserts into a larger piece of tissue. Abbreviated action potential duration was recorded at this junction, just proximal to the site of conduction block. This junction is analogous to the one where an accessory pathway inserts into the ventricle. Curved or fractionated atrial input into the accessory pathway, a poor source, may result in abbreviated action potential duration at the ventricular insertion site. Because the source-sink relationship predicts that success of ventricular preexcitation depends critically on the source at the ventricular insertion site, it follows that it also depends critically on the atrial input into the accessory pathway.

Wavefront collision and decreased safety factor for impulse propagation. Using segments of canine Purkinje fibers, Cranefield and Hoffman (6) demonstrated inhibition of impulse propagation at the center of the segment when wavefronts initiated from either end collided at the center. Other investigators have shown in canine ventricular muscle preparations abbreviated action potentials at the sites of wavefront collision $(7,8)$. The source-sink hypothesis would predict that at the sites of wavefront collision, fractionation of wavefronts with resultant shortened action potential duration and loss of electrotonic current would lead to decreased safety factor for impulse propagation. This is supported by previous in vitro data (6) and by our demonstration of two wavefronts colliding and inhibiting each other with resultant loss of accessory pathway conduction ( patient 1, Fig. 6).

Wavefront curvature. Collision of wavefronts may also result in local conduction block and shifting of wavefront direction and curvature (Fig. 7). In the spiral wave model of excitation, which is generic to many excitable media (9), the velocity of wavefront propagation depends on its curvature $(9,10)$. The sharper the curvature, the lower the velocity, until a minimum radius is reached and wavefront propagation can no longer sustain $(3,11)$. These data are consistent with the source-sink hypothesis in that as the curvature of a wavefront increases, a larger sink and a smaller source are generated as the result of the curvature. Lower source-sink ratio leads to lower safety factor for impulse propagation. Our findings in patient 5 (Fig. 7) demonstrated an association between wavefront curvature and loss of accessory pathway conduction and is compatible with the notion that increased curvature decreases the safety factor of impulse propagation (3). An additional observation in this patient was that the spatial geometry of wavefront curvature resembles that of a spiral wave of reentry, in which collision of wavefronts leads to curvature of wavefront propagation (10). Unfortunately, the plaque did not record the entire circuit of reentry to make a conclusive statement about the exact mechanism.

Wavefront summation. In addition to inhibition, summation of impulses has been described. Cranefield and Hoffman (6) using a segment of canine Purkinje fibers demonstrated that impulse propagation can occur at the center of a segment into an extension when the segment was stimulated simultaneously from both ends, whereas this propagation did not occur when either end was stimulated alone. The findings in patient 1 (Fig. 6 ), where two wavefronts arrived simultaneously and activated 
accessory pathway No. 1 (atrial wavefront No. 3, QRS No. 2) are consistent with summation. The previous in vitro data and our observations are readily explicable by the source-sink hypothesis. Critically timed arrival of two wavefronts may increase the current of the "source" and favor impulse propagation. How timing and relative direction of the two wavefronts determine whether summation or inhibition occurs is not known and should be the subject of future studies.

Wavefront breakup. In patient 2, spontaneous fractionation of atrial wavefronts near the accessory pathway was associated with loss of accessory pathway conduction and QRS transition (Fig. 8). Spontaneous breakup of spiral waves of reentrant excitation has been demonstrated in chemical reaction medium (9). This is intrinsic to spiral waves of excitation and occurs without external interferences (9). Depolarizing electrochemical energy would be expected to dissipate when wavefront fractionation occurs. This lowers the source-sink ratio and therefore the safety factor for propagation. An alternative explanation is that fractionated atrial electrograms, representing multiple small wavelets, may repetitively invade the accessory pathway and render it non-excitable by subsequent wavefronts (concealed conduction).

Antegrade concealed conduction. Inhibition of accessory pathway conduction during invasion or collision of wavefronts may represent a form of concealed conduction. It is possible that wavefronts that failed to activate the accessory pathway may have partially invaded the pathway, preventing subsequent excitation. Concealed antegrade conduction into the accessory pathway has been demonstrated with the atrial extrastimulus techniques during sinus rhythm, and has been proposed to explain the irregular pattern of preexcitation during atrial fibrillation $(12,13)$. However, concealed conduction during atrial fibrillation has not been shown. Our data showed that invasion by atrial wavefronts of low safety factor often resulted in subsequent loss of accessory pathway conduction and QRS transition (Figs. 4, 5, 6, and 8). These phenomena can be readily explained by the source-sink hypothesis. A wavefront with concealed conduction into the accessory pathway would render it relatively refractory. The next wavefront would then encounter partially refractory tissues and not be able to bring the accessory pathway to full excitation. The diminished output from this "source" would not be able to excite the larger sink, the ventricle, given the unfavorable geometry at the ventricular insertion site of the accessory pathway.

Retrograde conduction and concealed conduction. Theoretically, both retrograde conduction and retrograde concealed conduction may occur from the ventricles to the fibrillating atria via the accessory pathway, which may interfere with subsequent antegrade conduction from the atria to the ventricles. Retrograde concealed conduction in the preexcitation syndrome has been demonstrated using the extrastimulus techniques during sinus rhythm (12-15), and its role during atrial fibrillation has also been inferred (16). However, to date there has been no definitive demonstration of such in atrial fibrillation. In patient 1 with 2 accessory pathways, failure to activate accessory pathway No. 2 by atrial wavefront No. 3 (Fig. 6, panel 3) may be explained by retrograde concealment from accessory pathway No. 1 , as ventricular depolarization (electrode C8) from accessory pathway No. 1 arrived almost simultaneously and collided with atrial wavefront No. 3 (electrode B8).

Our data also showed an episode of retrograde invasion of the atrium during atrial fibrillation by wavefronts originating from atrioventricular node conduction (Fig. 5, panel 12), inhibiting accessory pathway activation by subsequent atrial wavefronts (Fig. 5, panel 13). This added atrial input may complicate the patterns of activation during atrial fibrillation, contributing to the maintenance of this arrhythmia. These findings confirmed a role of retrograde overt and concealed conduction in maintaining consecutively non-preexcited QRS complexes during atrial fibrillation (16), and may partially explain the prevention of sustained atrial fibrillation after successful surgical ablation of the accessory pathways $(17,18)$. Further studies on how new atrial wavefronts may be generated by retrograde conduction during atrial fibrillation may improve our understanding on how human atrial fibrillation is generated and maintained.

The importance of the accessory pathway effective refractory period. Wellens et al. (19) demonstrated a strong relationship between accessory pathway refractory period and the shortest $R R$ interval during atrial fibrillation. One might expect that transition of QRS morphology may be due to the arrival of an atrial wavefront within the refractory period of the accessory pathway, allowing time for conduction through the atrioventricular node to occur. However, this was the case in only two episodes of QRS transition. In these cases, the timing of atrial input appeared to "dissociate" from the ventricle and "march" into the accessory pathway refractory period with resultant loss of preexcitation (Fig. 9). Although the accessory pathway refractory period is an important determinant of accessory pathway conduction, it does not explain most episodes of accessory pathway conduction block among those patients studied (Fig. 10 ). In the majority of cases, changes in spatial characteristics of atrial wavefronts which lowered the source-sink ratio preceded the loss of accessory pathway conduction. It is worth mentioning that the refractory period is not a consideration of propagation properties, but of repolarization properties. Furthermore, our data primarily support the importance of the source current in determining wavefront propagation. The conduction properties of the sink (the accessory pathway and the ventricle) cannot be determined from our data and does not emerge as an important factor.

Implications. Atrial fibrillation remains an important cardiovascular problem (20). Although functional reentry is believed to underlie atrial fibrillation, the exact mechanism for its genesis and maintenance remains unclear. Mapping of human atrial fibrillation demonstrated the presence of multiple reentrant wavefronts $(5,21)$. Our study is compatible with these findings. In addition, we showed that safety factor for wavefront propagation in humans depends on the characteristics of the source wavefront. Wavefront curvature, breakup, and collision in the atrium resulted in a lower safety factor for impulse propagation through the accessory pathway. A broader implication of these findings is that, because the source-sink relationship is important in determining the safety factor for impulse propagation, it follows that wavefront curvature may play an important role in the propagation of cardiac impulses (3) and contribute to the generation and maintenance of functional reentry in humans.

Limitations. Due to the limited resolution of our mapping system, the presence of fat in the atrioventricular groove, and the absence of endocardial recording, accessory pathway potentials were not consistently recorded. Therefore, the exact site of conduction block among atrial wavefronts that failed to activate the accessory pathway cannot be determined. It is possible that conduction block occurred at the accessory pathway atrial insertion site, ventricular insertion site, or within the pathway itself. 
However, this limitation would not affect the main conclusion of this study, that local atrial wavefront characteristics are important factors influencing impulse propagation from the atria to the ventricles via the accessory pathway. Because of limited view of the recording electrode array, wavefronts originating from outside the mapping area could not be seen and their effects on accessory pathway conduction could not be determined. Also, simultaneous recording of atrial wavefronts at the atrioventricular node was not performed and their roles in the transition of QRS morphology could not be assessed.

Conclusion. We have demonstrated that the safety factor for impulse propagation during atrial fibrillation depends on the wavefront characteristics. In the in situ human heart, spatially uniform atrial wavefronts are more likely to result in accessory pathway activation while invasion by secondary wavefronts often results in collision and marked curvature or fractionation of wavefronts. This leads to subsequent loss of accessory pathway conduction. These observations are consistent with the sourcesink hypothesis on the safety factor for impulse propagation in the human heart.

\section{Acknowledgments}

We thank Dr. James Forrester for his support, Dr. Timothy A. Denton for his statistical assistance, and Elaine Lebowitz and Kerry McElhaney for their secretarial assistance.

This work was done during the tenure of an American College of Cardiology/Merck Fellowship Award to J. J. C. Ong and an American Heart Association/Wyeth-Ayerst Established Investigatorship Award (93002670) to P.-S. Chen, and was supported in part by a National Institutes of Health FIRST Award (HL-50259), a National Institutes of Health SCOR grant (HL-52319), and an American Heart Association National Center Grant-in-Aid (92009820).

\section{References}

1. Mendez, C., W. J. Mueller, and X. Urguiaga. 1970. Propagation of impulses across the purkinje fiber-muscle junctions in the dog heart. Circ. Res. 26:135150 .

2 de la Fuente, D., B. Sasyniuk, and G. K. Moe. 1971. Conduction through a narrow isthmus in isolated canine atrial tissue. Circulation. 44:803-809.

3. Cabo, C., A. M. Pertsov, W. T. Baxter, J. M. Davidenko, R. A. Gray, and
J. Jalife. 1994. Wavefront curvature as a cause of slow conduction and block in isolated cardiac muscle. Circ. Res. 75:1014-1028.

4. Chen, P.-S., W. P. Dembitsky, R. P. Fleck, C. M. Calisi, and G. K. Feld. 1990. Demonstration of accessory pathway interaction by computerized mapping in preexcitation syndrome. PACE. 13:839-844.

5. Konings, K. T. S., C. J. H. J. Kirchhof, J. R. L. M. Smeets, H. J. J. Wellens, O. C. Penn, and M. A. Allessie. 1994. High-density mapping of electrically induced atrial fibrillation in humans. Circulation. 89:1665-1680.

6. Cranefield, P. F., and B. F. Hoffman. 1971. Conduction of the cardiac impulse. Circ. Res. 28:220-233.

7. Toyoshima, H., and M. J. Burgess. 1978. Electrotonic interaction during canine ventricular repolarization. Circ. Res. 43:348-356.

8. Osaka, T., I. Kodama, N. Tuboi, J. Toyama, and K. Yamada. 1987. Effects of activation sequence and anisotropic cellular geometry on the repolarization phase of action potential of dog ventricular muscles. Circulation. 76:226-236.

9. Gerhardt, M., H. Schuster, and J. J. Tyson. 1990. A cellular automation model of excitable media including curvature and dispersion. Science (Wash. DC). 247:1563-1566

10. Foerster, P., S. C. Muller, and B. Hess. 1988. Curvature and propagation velocity of chemical waves. Science (Wash. DC). 241:685-688.

11. Foerster, P., S. C. Muller, and B. Hess. 1989. Critical size and curvature of wave formation in an excitable chemical medium. Proc. Natl. Acad. Sci. USA. 86:6831-6834.

12. Klein, G. J., R. Yee, and A. D. Sharma. 1984. Concealed conduction in accessory atrioventricular pathways: an important determinant of the expression of arrhythmias in patients with Wolff-Parkinson-White syndrome. Circulation. 70:402-411.

13. Svinarich, J. T., D. Y. Tai, J. Mickelson, E. C. Keung, and R. J. Sung. 1985. Electrophysiologic demonstration of concealed conduction in anomalous atrioventricular bypass tracts. J. Am. Coll. Cardiol. 5:898-903.

14. Zipes, D. P., R. L. DeJoseph, and D. A. Rothbaum. 1974. Unusual properties of accessory pathways. Circulation. XLIX:1200-1211.

15. Prystowsky, E. N., E. L. C. Pritchett, and J. J. Gallagher. 1984. Concealed conduction preventing anterograde preexcitation in Wolff-Parkinson-White syndrome. Am. J. Cardiol. 53:960-961.

16. Chen, P.-S., and E. N. Prystowksy. 1991. Role of concealed and supernormal conductions during atrial fibrillation in the preexcitation syndrome. Am. J. Cardiol. 68:1329-1334.

17. Sharma, A. D., G. J. Klein, G. M. Guiraudon, and S. Milstein. 1985. Atrial fibrillation in patients with Wolff-Parkinson-White syndrome: incidence after surgical ablation of the accessory pathway. Circulation. 72:161-169.

18. Chen, P-S., J. C. Pressley, A. S. L. Tang, D. L. Packer, J. J. Gallagher, and E. N. Prystowsky. 1992. New observations on atrial fibrillation before and after surgical treatment in patients with the Wolff-Parkinson-White syndrome. $J$. Am. Coll. Cardiol. 19:974-981.

19. Wellens, H. J. J. and D. Durrer. 1974. Wolff-Parkinson-White syndrome and atrial fibrillation: relation between refractory period of accessory pathway and ventricular rate during atrial fibrillation. Am. J. Cardiol. 34:777-782.

20. Wellens, H. J. J. 1994. Atrial fibrillation-the last big hurdle in treating supraventricular tachycardia. N. Engl. J. Med. 331:944-945.

21. Cox, J. L., T. E. Canavan, R. B. Schuessler, M. E. Cain, B. D. Lindsay, C. Stone, P. K. Smith, P. B. Corr, and J. B. Boineau. 1991. The surgical treatment of atrial fibrillation. II: Intraoperative electrophysiologic mapping and description of the electrophysiologic basis of atrial flutter and atrial fibrillation. J. Thorac. Cardiovasc. Surg. 101:406-426. 